\title{
$\mathrm{BaTiO}_{3}$ 半导体陶瓷从 PTC 特性向边界层 电容效应过渡问题探讨 晶界势垒模型
}

\author{
郑振华 缪容之陈 羽
}

(宁波大学物理系, 宁波 315211)

摘要

本文分析了 $\mathrm{BaTiO}_{3}$ 半导体陶瓷的 Daniels 模型存在的问题, 提出了从 PTC 特性 向边界层电容效应过渡的晶界势垒模型, 解决了 Daniels 模型的缺陷, 使 $\mathrm{BaTiO}_{3}$ 半 导瓷及其有关器件的物理性能的定量计算成为可能.

\section{关链词 $\mathrm{BaTiO}_{3}$ 半导体陶瓷、PTC 特性、边界展电容效应、晶界势垒模型}

\section{1 引 言}

$\mathrm{BaTiO}_{3}$ 陶瓷的半导体化研究, 导致了 1955 年半导体化 $\mathrm{BaTiO}_{3}$ 陶瓷的 $\mathrm{PTC}$ 特性的发现 ${ }^{[1]}$. 之后在 60 和 70 年代又成功地研究出了半导体化 $\mathrm{BaTiO}_{3}$ 陶瓷边界层电容器 ${ }^{[2]}$ 和 $\mathrm{SrTiO}_{3}$ 边界 层电容器 ${ }^{[3]}$, 由于它的优越性能, 已经有了广泛的应用. 对半导体化 $\mathrm{BaTiO}_{3}$ 陶瓷有关性能的 理论解释, 60 年代初, Heywang ${ }^{[\mathrm{f}}$ 提出了 PTC 特性的晶界势垒模型, 而后 Jonker ${ }^{[\sqrt{5}}$ 对 Heywang 模型增加了铁电补偿修正, Heywang 和 Jonker 等人的理论较好地解释了部分实验现象, 但仍 存在许多问题. 在 Heywang 模型的基础上, 1976 年 Daniels 等人间通过详细的缺陷理论研 究, 提出了钡缺位模型, 试图将半导体化 $\mathrm{BaTiO}_{3}$ 陶瓷的 PTC 特性和边界层电容效应统一于一 个模型, 这一模型的提出解决了许多半导体化 $\mathrm{BaTiO}_{3}$ 陶瓷的遗留问题, 得到了人们的重视.

然而, 根据我们在本文的探讨, Daniels 等人的钡缺位模型在解释半导体化 $\mathrm{BaTiO}_{3}$ 陶瓷从 PTC 特性向边界层电容效应过渡的电阻率巨变问题和高介电常数引起的载流子耗尽层厚度 问题时存在困难, 并且从 Daniels 模型不能定量计算出 PTC 特性向边界层电容效应过渡的有 关物性参数, 说明 Daniels 等人的钡缺位模型需要补充或修正.

本文首先探讨 Daniels 等人的钡缺位模型存在的问题, 在详细的晶界受主态的形成和本 质的探讨基础上, 提出了同时存在晶界受主态和钡缺位扩散层下的势垒模型. 


\section{Daniels 模型存在的问题}

\section{1 晶界势垒的宽度问题}

图 1 为 Daniels 等人 ${ }^{[0.7}$ 在解释 $\mathrm{BaTiO}_{3}$ 半导瓷从 PTC 特性向边界层电容效应过渡时提出

(a) $l_{\mathrm{D}}>l_{\mathrm{B}}$

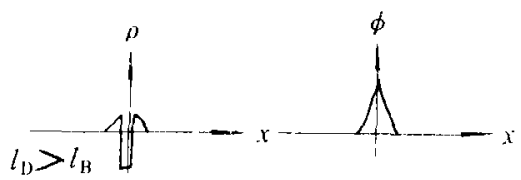

(b)

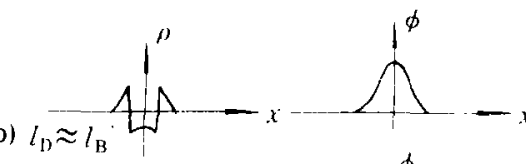

(c)

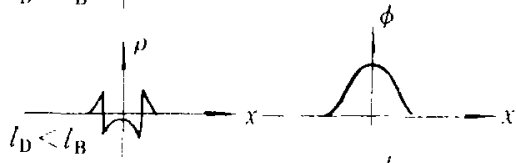

(d) $l_{\mathrm{D}} \ll l_{\mathrm{B}}$

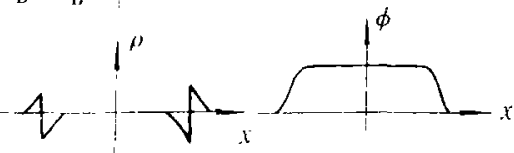

图 1 Daniels 模型的电荷分布 $($ 左 $)$ 和势垒 (右) $)^{[6,7]}$

$l_{\mathrm{D}}$ 为载流子耗尽层厚度, $l_{\mathrm{B}}$ 为钡缺位扩散层厚度 的钡缺位模型的示意图, 其中 (a) 为 PTC 特性, (d) 为 边界层电容效应, (b), (c) 为两者的过渡状态, 图中的 势垒高度约为 $1.0 \mathrm{eV}$. 然而由于铁电性 $\mathrm{BaTiO}_{3}$ 半导 瓷具有很高的介电常数, 图 1 所示的势垒模型将受严 重影响, 由晶界的双 Schottky 模型, 得到耗尽层近似 下晶界势垒的表达式为 ${ }^{[4]}$

$$
\varphi=\frac{\mathrm{e}^{2} N_{\mathrm{s}}^{2}}{8 \varepsilon_{0} \varepsilon_{\text {eff }} N_{\mathrm{D}}}=\frac{\mathrm{e}^{2} N_{\mathrm{s}} l_{\mathrm{D}}}{4 \varepsilon_{0} \varepsilon_{\text {eff }}}=\frac{\mathrm{e}^{2} N_{\mathrm{D}} l_{\mathrm{D}}^{2}}{2 \varepsilon_{0} \varepsilon_{\text {eff }}},
$$

式中 $N_{\mathrm{S}}$ 为晶界受主态密度, $N_{\mathrm{D}}$ 为晶粒的施主浓度, $l_{\mathrm{D}}$ 为施主耗尽层厚度, $\varepsilon_{\mathrm{eff}}$ 为有效介电常数, $\mathrm{e}$ 为电子电 荷, $\varepsilon_{0}$ 为真空介电常数. 在 $\mathrm{BaTiO}_{3}$ 半导瓷中 $\varepsilon_{\mathrm{eff}}$ 达 $10^{4}$,

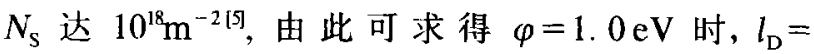
$2.2 \times 10^{-6} \mathrm{~m}$.

对于介电常数达 $10^{5}$ 数量级的边界层电容器,晶 粒大小在数十微米量级, 相应的晶界高阻层在微米量 级, 而每个晶粒有两个 $n n^{+}$结或 Schottky 结, 结区的 总厚度在 $5-10 \mu \mathrm{m}$ 范围, 这样晶界高阻层全部都是 载流子耗尽层. 图 1 中 (d) 的情况不存在, 其它情况不准确甚至不成立.

\section{2 电阻率变化问题}

$\mathrm{BaTiO}_{3}$ 半导瓷是多晶材料, 应满足多晶材料的电阻率和介电常数的色散关系, 根据 Koops 等人的结果 ${ }^{[8,9]}$, 直流情况电阻率的表达式为

$$
\rho \doteq \rho_{\mathrm{G}}+\alpha \rho_{\mathrm{B}} \doteq \alpha \rho_{\mathrm{B}}=d \rho_{\mathrm{B}} / D,
$$

式中 $\rho_{\mathrm{G}}$ 和 $\rho_{\mathrm{B}}$ 分别为晶粒和晶界高阻层的电阻率, $d$ 为晶界高阻层的总厚度, $D$ 为晶粒尺寸, $\alpha=d / D$ 为晶界高阻层的厚度分数.

如果根据'Daniels 模型, 在不同厚度的钡缺位扩散层时具有相同的势垒高度, 亦即晶界高 阻层具有相同的电阻率, 则由 (2) 式可知此时的电阻率和晶界高阻层的厚度尔数成正比. 对 于一般情况, 在 PTC 特性时高阻层的厚度为 $10^{2}-10^{3} \AA$, 而在边界层电容效应时厚度为 $10^{3}-10^{4} \AA$, 晶界高阻层的厚度增加 $10^{1}-10^{2}$ 倍, 而实际情况从 PTC 特性向边界层电容效应 过渡时的电阻率变化在 $10^{8}-10^{10}$ 倍. 两者的差距如此之大, 不可能从图 1 所示的势垒模型中 按 $n n^{+}$结或双 Schottky 结对电阻率的贡献得到解释。

\section{3 定量计算问题}

Daniels 等人的钡缺位模型, 虽然清楚地描述了半导体化 $\mathrm{BaTiO}_{3}$ 陶瓷从 PTC 特性向边界 层电容效应过渡的物理图象, 但这一模型对 $\mathrm{BaTiO}_{3}$ 半导瓷从 PTC 特性向边界层电容效应过 渡只能作出定性的解释, 不能由此模型定量计算出 PTC 特性和边界层电容效应特别是两者的 
中间状态的有关物理性能参数. 但定量的结果无论对理论模型本身还是对有关性能的器件的 设计和制造都是很重要的, 并且根据上述对 Daniels 模型不足的分析, 表明 Daniels 等人的钡 缺位模型需要进一步的完善。

\section{3 晶界受主态及其本质探讨}

对 $\mathrm{BaTiO}_{3}$ 半导体陶瓷，晶界受主态是 PTC 特性是否存在和特性强弱的关键因素．对边 界层电容效应, 如果根据 Daniels 等人的模型, 不但晶界受主态已经从 PTC 特性时的二维晶 界扩展成三维空间, 而且由于 $n n^{+}$结的存在使晶界受主态失去了作用. 然而, 根据上一节的分 析, 实际的钡缺位扩散层厚度不能达到 Daniels 等人认为的厚度, 说明晶界受主态在边界层电 容效应情况仍有作用,下面先对此进行探讨.

根据 Tamm 和 Shockley 的表面态理论 ${ }^{[10]}$, 当原子的周期规则排列在固体表面消失后, 固 体在原有的能带上出现了新的许可能级一一表面电子态, 对于半导体和绝缘体, 表面电子态 的能级位于禁带中.

对于多晶材料的晶粒间界, 由于原子周期排列的消失, 在晶界也应出现新的许可能级, 即晶 界电子态. 但由于在晶界出现的只是化学组分和原子结构等的变化, 晶界电子态应具有和表 面不同的特点, 这些特点必然和晶界的化学组分, 原子或离子的状态和结构、缺陷及其分布等 因素有关。

根据热力学和统计物理理论, 由于杂质原子和缺陷在晶粒内部和晶界具有不同的自由 能, 使得杂质和缺陷在晶界偏析, 从而导致晶粒内部和晶界具有不同的化学组分. 化学组分的 差别又导致原子或离子结构的不同, 结构和化学组分的差别使得晶界电子态也不相同. 对于 半导体陶瓷, 由上述化学组分和无序结构形成的晶界电子态位于禁带中, 相应的能级低于晶 粒内部的 Fermi能级.

由于陶瓷烧结的冷却过程是一个非平衡热力学过程, 冷却过程中的新状态首先在原子结 构比较松散, 原子扩散比较容易的晶界建立, 然后逐渐向晶粒内部移动. 因此; 晶界的化学组 分和原子结构与冷却速度和冷却气氛密切相关. 对于通常较高氧分压气氛的冷却过程, 高温 烧结时的氧平衡状态被破坏后,通过氧离子的扩散首先在晶界形成过氧化状态, 晶界的氧离 子浓度增加导致晶界形成正离子缺位、过氧化态离子特别是过氧化态杂质离子以及直接的吸 附氧离子. 在继续的冷却过程中, 晶界进一步被氧化, 形成更大数量的正离子缺位、过氧化态 离子和吸附氧离子, 并且, 这些过氧化态缺陷逐步向晶粒内部扩散, 在有限的冷却速度下在晶 粒表面形成正离子缺位和吸附或间隙氧离子为主的 (由于偏析, 杂质离子更难进人晶粒)扩散 层或过氧化状态层. 晶界附近的过氧化状态及其在晶粒表面的扩散层在冷却过程中不断增 长, 直至降温到离子很难移动, 结构被冻结的温度. 对于 $\mathrm{BaTiO}_{3}$ 半导体陶瓷, 晶粒表面的过 氧化状态层就是 Daniels 等人指出的钡缺位扩散层 ${ }^{[a, 7}$, 尽管扩散层中也有少量的间隙氧离子 和钝离子缺位等其它过氧化态缺陷.

晶界电子态的性质很大程度上是由晶界中离子所处的状态尤其是氧化或还原程度决定 的. 从定性上说明, 当晶界处于过氧状态时, 晶界形成的正离子缺位、过氧化状态离子和吸附 氧离子都表现为受主特性, 相应的能级位于能带中禁带的下半部分, 并且氧化程度越高, 所处 的能级越低或者能态密度越高, 相反, 当晶界处于还原或缺氧状态时, 晶界中缺陷和离子都表 
现为施主特性, 相应的能级位于禁带的上半部分, 并且还原程度越高, 所处的能级越高. 晶界 电子态的能级高低决定了 $\mathrm{BaTiO}_{3}$ 半导瓷 PTC 特性的强弱, 这也就是还原气氛烧成的 $\mathrm{BaTiO}_{3}$ 半导瓷没有或只有很弱的 PTC 特性的原因.

因此, 晶界受主态不但是因周期性原子排列消失而形成的、能级低于晶粒的 Fermi 能级 的、相对于唱粒的 Fermi 能级表现为受主特性的晶界电子态; 而且更重要的是晶界中由正离 子缺位、过氧化态离子和吸附氧离子决定的, 表现为受主性质的深能级晶界电子态。在非平 衡的冷却过程中, 虽然首先在晶界形成的过氧化状态不断向晶粒内部扩散而形成过氧化状态 扩散层, 作为扩散的源, 晶界的氧化状态肯定比扩散层的高或者晶界的正离子缺位, 过氧化态 离子和吸附氧离子的浓度肯定高于扩散层中的浓度. 因此, 在任何冷却条件得到的半导瓷, 唱 界受主态总是存在的, 其能级低于扩散层的能级, 其能态密度高于扩散层的能态密度.

晶界受主态的能级和密度与冷却速度的关系, 冷却速度快时妨碍氧离子扩散进人陶瓷内 部的晶界, 也妨碍晶界的过氧化状态扩散进人晶粒, 故只有表面附近的晶界受主态能级低和 密度高, 陶瓷内部难以形成低能级高密度的晶界受主态. 冷却速度慢时晶界的过氧化态扩散 进人晶粒的多, 晶界受主态因此而能级上升密度降低. 晶粒表面的过氧化状态扩散层 $\left(\mathrm{BaTiO}_{3}\right.$ 中的饮缺位扩散层) 的厚度由冷却速度决定, 冷却速度越慢, 扩散层的厚度越厚。扩散层中过 氧化态缺陷的浓度和载流子的浓度中冷却气氛决定, 气氛中氧分压越高, 扩散层中的缺陷浓 度越高相应的载流子浓度越低或扩散层中的能级越低。

对于还原气氛烧成的半导瓷作氧化气氛的热处理的情况, 晶界受主态和过氧化态扩散层 的形成和性质有与上述讨论相似的结果, 在此不再作详细的讨论.

\section{4 势 垒 模 型}

根据 Heywang 的讨论 ${ }^{[4]}$, 晶界高阻层的电阻率和它的势垒的关系为

$$
\rho_{\mathrm{B}}=\rho_{0} \exp (\varphi / k T),
$$

式中 $\rho_{0}$ 为势垒 $\varphi=0$ 时高阻层的电阻率, $\varphi$ 为高阻层的势垒高度, $T$ 为温度, $k$ 为 Boltzmann 常 数.

由 (3) 式可知, 当 $\mathrm{BaTiO}_{3}$ 半导瓷从 PTC 特性问边界层电容效应过渡时, 晶界势垒必定增 加了 $1.0 \mathrm{eV}$ 左右, 这一势垒高度的增加是由钡缺位扩散层厚度的增加引起的. 同时, 根据上一 节的探讨, 钡缺位扩散层形成后晶界受主态仍然存在. 因此, 必须在同时存在晶界受主态和 钡缺位扩散层的条件下建立从 PTC 特性向边界层电容效应过渡的势垒模型。

\section{1 势垒模型}

图 2 为同时存在晶界受主态和钡缺位扩散层时的晶界势垒模型的示意图, 其中图 2(a) (c) 表示钡缺位扩散层的厚度增加使得晶界势垒高度增加. 当势垒高度增加到 $\left(E_{\mathrm{F}}-E_{\mathrm{b}}\right)\left(E_{\mathrm{b}}\right.$ 为 晶界受主态的能级) 时, 势垒高度不再增加, 但势垒形状发生变化. 对于不同的晶界受主态能 级, 示意图为图 2(d) 和 (e), 其中 (d) 为晶界受主态的能级等于或高于钡缺位扩散层的能级的 情况, (e) 为晶界受主态的能级低于钡缺位扩散层的能级的情况.

对于晶界势垒小于饱和值或极限值 $\left(E_{\mathrm{F}}-E_{\mathrm{b}}\right)$ 的情况, 可求得耗尽层近似下图 2 的势垒模 型的晶界势垒的表达式为 
(a) $l_{\mathrm{B}} \sim 0$

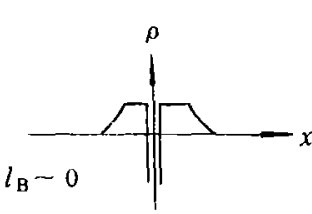

(b)
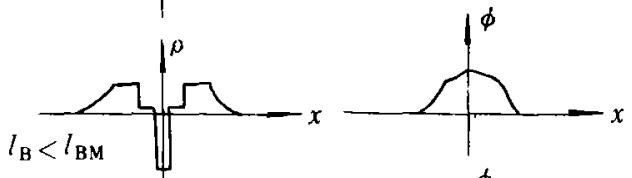

(c)
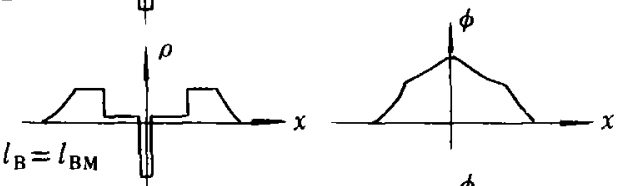

(d)
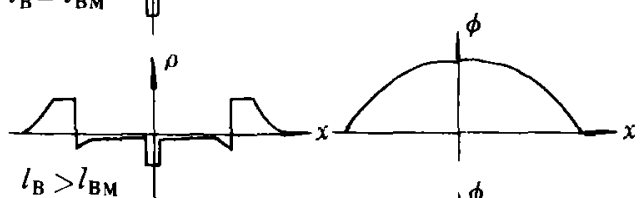

(e) $l_{\mathrm{B}}>l_{\mathrm{BM}}$

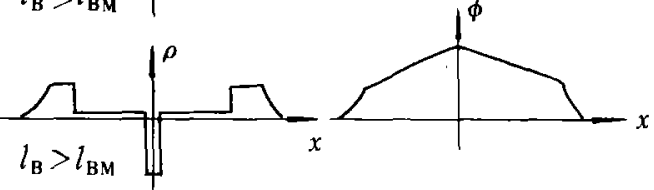

图 2 晶界势垒模型

左图为电荷分布, 右图为势垒; $l_{\mathrm{B}}$ 为钡缺位扩散层的厚度, $l_{\mathrm{BM}}$ 为势垒刚达到饱和时的 $l_{\mathrm{B}}$

$$
\varphi=\frac{\mathrm{e}^{2}}{2 \varepsilon_{0} \varepsilon_{\mathrm{eff}}}\left(N_{\mathrm{D}} l_{\mathrm{D}}^{2}+2 N_{\mathrm{D}} l_{\mathrm{D}} l_{\mathrm{B}}+N_{\mathrm{B}} l_{\mathrm{B}}^{2}\right),
$$

式中 $N_{\mathrm{B}}$ 为钡缺位扩散层中形成势垒后的正 电荷密度, $l_{\mathrm{B}}$ 为钡缺位扩散层的厚度.

根据电荷守恒关系式:

$$
N_{\mathrm{S}}=2\left(N_{\mathrm{D}} l_{\mathrm{D}}+N_{\mathrm{B}} l_{\mathrm{B}}\right),
$$

代人 (4) 式得

$$
\begin{aligned}
\varphi & =\frac{\mathrm{e}^{2}}{4 \varepsilon_{0} \varepsilon_{\text {eff }}}\left[N_{\mathrm{S}}\left(l_{\mathrm{D}}+2 l_{\mathrm{B}}\right)-2 N_{\mathrm{B}} l_{\mathrm{B}}\left(l_{\mathrm{D}}+l_{\mathrm{B}}\right)\right] \\
& =\frac{\mathrm{e}^{2}}{4 \varepsilon_{0} \varepsilon_{\text {eff }}}\left[N_{\mathrm{S}} l_{\mathrm{B}}+2 N_{\mathrm{D}} l_{\mathrm{D}}\left(l_{\mathrm{D}}+l_{\mathrm{B}}\right)\right] .
\end{aligned}
$$

由于晶界受主态的能级的限制, (4) 和 (6) 式 的势垒高度的极限值或饱和值为 $\left(E_{\mathrm{F}}-E_{\mathrm{b}}\right)$. 由 (6) 式可求得晶界势垒达到饱和值时的铞 缺位扩散层的厚度 $l_{\mathrm{BM}}$ 为

$$
l_{\mathrm{BM}}=\frac{4\left(E_{\mathrm{F}}-E_{\mathrm{b}}\right) \varepsilon_{0} \varepsilon_{\mathrm{eff}} / \mathrm{e}^{2}-2 N_{\mathrm{D}} l_{\mathrm{D}}^{2}}{N_{\mathrm{s}}+2 N_{\mathrm{D}} l_{\mathrm{D}}} .
$$

当钡缺位扩散层的厚度增加到大于 $l_{\mathrm{BM}}$ 时, 为使晶界势垒的高度保持不变, 晶界受主 态的填充率下降以平衡由于钡缺位扩散层厚 度增加引起的电场区的增宽, 这样也导致了晶界势垒形状的变化. 对图 2(d) 的晶界受主态 的能级高于或等于钡缺位扩散层的能级的情况, 晶界势垒将逐渐转变为双 $n^{+} n$ 结. 对图 2(e) 的晶界受主态的能级低于钡缺位扩散层的能级的情况, 晶界受主态的作用不会消失, 晶界势 垒由图 2(c) 的较尖的形状逐渐变得平坦.

\section{2 钡缺位扩散层中的电荷及其对势垒的影响}

根据 (6) 式, 钡缺位扩散层中的电荷降低晶界势垒和电阻率, 而钡缺位扩散层中的电荷分 布是由晶界受主态和钡缺位扩散层的相对能级决定的, 晶界受主态和钡缺位扩散的能级是由 冷却速度和冷却气氛决定的, 因此有必要结合具体的能带结构和冷却条件分析钡缺位扩散层 中可能出现的电荷分布.

根据图 3 所示的能带图 ${ }^{[11]}$, 对不同的氧分压气氛形成的钡缺位扩散层的电荷分布主要有

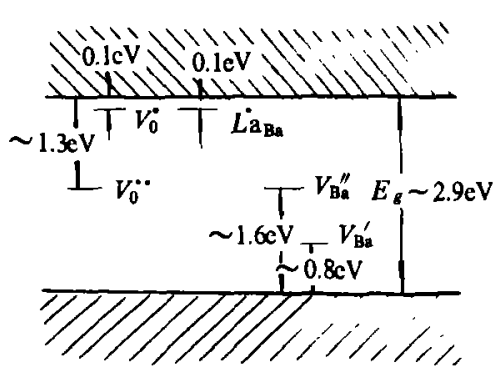

图 $3 \mathrm{BaTiO}_{3}$ 的能带略图 两种情况: 第一种情况为晶界受主态的能级和钡缺位扩散 层的能级均介于 $L a_{\mathrm{Ba}}^{*}$ 和 $V_{\mathrm{Ba}}^{\prime \prime}$ 的能级之间(一般氧分压情 况), 由于 $L a_{\mathrm{Ba}}^{*}$ 和 $V_{\mathrm{Ba}}^{\prime \prime}$ 之间没有能级, 钡缺位扩散层中没有高 于晶界受主态能级的载流子去填充晶界受主态, 故此时钡 缺位扩散层中没有净电荷分布. 第二种情况为钡缺位扩散 层中的能级高于或等于 $V_{\mathrm{Ba}}^{\prime \prime}$ 的能级, 而晶界受主态的能级 低于 $V_{\mathrm{Ba}}^{\prime \prime}$ 的能级 (高氧分压气氛情况), 由于只有 $V_{\mathrm{Ba}}^{\prime \prime}$ 能级上 的载流子可以填充晶界受主态, 使得钡缺位扩散层有密度 
恒为 $V_{\mathrm{Ba}}^{\prime \prime}$ 能级密度的电荷分布. 对于更深的能级, 也可以得到相应的电荷分布, 但一般不可能 出现. 事实上, 对晶界势垒高度 $\varphi=0-1.2 \mathrm{eV}\left(\mathrm{La}_{\mathrm{B}_{2}}^{*}\right.$ 和 $V_{\mathrm{Ba}}^{\prime \prime}$ 的能级差 $)$ 都在上述第一种情况的 范围, 实验上也未见有势垒高度大于 $1.2 \mathrm{eV}$ 的报道.

对于上述的第一种情况, 有 $N_{\mathrm{B}} \sim 0$, 此时 (6) 式的晶界势垒表达式化为

$$
\begin{aligned}
\varphi & =\frac{\mathrm{e}^{2} N_{\mathrm{D}}}{2 \varepsilon_{0} \varepsilon_{\text {eff }}} l_{\mathrm{D}} \cdot\left(l_{\mathrm{D}}+2 l_{\mathrm{B}}\right)=\frac{\mathrm{e}^{2} N_{\mathrm{S}}}{4 \varepsilon_{0} \varepsilon_{\mathrm{eff}}}\left(l_{\mathrm{D}}+2 l_{\mathrm{B}}\right) \\
& =\frac{\mathrm{e}^{2} N_{\mathrm{S}}^{2}}{8 \varepsilon_{0} \varepsilon_{\mathrm{eff}} N_{\mathrm{D}}}\left(1+2 \frac{l_{\mathrm{B}}}{l_{\mathrm{D}}}\right) .
\end{aligned}
$$

而 (7) 式化为

$$
l_{\mathrm{BM}}=\frac{2 \varepsilon_{0} \varepsilon_{\mathrm{eff}}\left(E_{\mathrm{F}}-E_{\mathrm{b}}\right)}{\mathrm{e}^{2} N_{\mathrm{S}}}-\frac{l_{\mathrm{D}}}{2}=\frac{\varepsilon_{0} \varepsilon_{\mathrm{eff}}\left(E_{\mathrm{F}}-E_{\mathrm{b}}\right)}{\mathrm{e}^{2} N_{\mathrm{D}} l_{\mathrm{D}}}-\frac{l_{\mathrm{D}}}{2} .
$$

\section{3 物理性能参数的计算公式}

由多晶材料的电阻率和介电常数的色散关系的结果 ${ }^{[8,9]}$ 得到电阻率和介电常数的色散关 系为

$$
\begin{gathered}
\rho=\rho_{\mathrm{G}}+\alpha \rho_{\mathrm{B}} /\left(1+\omega^{2} \tau_{\rho}^{2}\right), \\
\varepsilon=\varepsilon_{\mathrm{G}}+\left(\frac{\varepsilon_{\mathrm{B}}}{\alpha}-\varepsilon_{\mathrm{G}}\right) /\left(1+\omega^{2} \tau_{\varepsilon}^{2}\right),
\end{gathered}
$$

式中 $\varepsilon_{\mathrm{G}}$ 和 $\varepsilon_{\mathrm{B}}$ 分别为晶粒和晶界高阻层的介电常数, $\omega$ 为角频率, 弛豫时间 $\tau_{\rho}=\varepsilon_{0} \varepsilon_{\mathrm{B}}\left(\rho_{\mathrm{G}} \rho_{\mathrm{B}} / \alpha\right)^{1 / 2}$, $\tau_{\varepsilon}=\varepsilon_{0} \varepsilon_{\mathrm{B}} \rho_{\mathrm{G}} / \alpha$. 对于一般情况恒有 $\alpha \ll 1, \rho_{\mathrm{B}} \gg \rho_{\mathrm{G}}$ 以及 $\alpha \rho_{\mathrm{B}} \gg \rho_{\mathrm{G}}$.

由 (11) 式可得到直流或低频情况的介电常数为

$$
\varepsilon=\varepsilon_{\mathrm{B}} / \alpha=D \varepsilon_{\mathrm{B}} / d .
$$

根据前面的讨论, 低频或直流情况的电阻率表达式分别为 (2)和 (3) 式, 其中势垒高度对不 同的情况分别为 (6) 和 (8) 式, 势垒的上限为 $\varphi=E_{\mathrm{F}}-E_{\mathrm{b}}$. 由 (10) 和 (11) 式结合 (3) 式的电阻率 和介电常数可以得到介电损耗因子 $\operatorname{tg} \delta$ 为

$$
\operatorname{tg} \delta=(\omega C R)^{-1}=\left(\omega \varepsilon_{0} \varepsilon \rho\right)^{-1},
$$

式中 $C$ 和 $R$ 分别为被测样品的电容和电阻.

此外, 由电阻率的表达式还可以求得 PTC 特性有关的升阻比和电阻率温度系数等参数.

\section{5 结 论}

本文建立了 $\mathrm{BaTiO}_{3}$ 半导体陶瓷从 PTC 特性向边界层电容效应过渡的晶界势垒模型, 由 该模型可以得到 $\mathrm{BaTiO}_{3}$ 半导瓷的 PTC 特性和边界层电容效应及其任何中间状态的物理性能 参数的定量结果. 本文的模型解决了 Daniels 等人提出的钡缺位模型存在的问题, 也为 $\mathrm{BaTiO}_{3}$ 半导瓷及其有关器件的设计提供了理论基础. 模型的详细结果及其对 $\mathrm{BaTiO}_{3}$ 半导瓷的 PTC 电阻器和边界层电容器设计的应用将在其它文章中讨论.

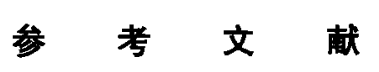

[1] Haayman, P. W. et al., German Patent 929350, 1955. 
[2] Waku, S., Rev. Electr. Comm. Lab., 1967, 15:689.

[3] Edahiro, T., Yoshimura, F., Rev. Electr. Comm. Lab., 1973, 21:843.

[4] Heywang, W., Solid State Electronics, 1961, 3:51.

[5] Jonker, G. H., Solid State Electronics, 1964, 7:895.

[6] Daniels, J., Wernicke, R., Philips Res Rept., 1976, 31:544.

[7] Daniels, J., Härdtl, K. H., Wernicke, R., Philips Tech. Rev., 1978/79, 38:73.

[8] Koops, C. G., Phys. Rev., 1951, 83:121.

[9] Stratte, T. G., Adv. in Ceramics, 1986, 7:114.

[10] Mark, P., Bottoms, W. R., Progress in Solid State Chemistry, Vol. 6, 17.

[11] Daniels, J., Härdtl, K. H., Philips Res. Rept., 1976, 31:489. 